\title{
A Novel Protocol for Medical Management of Cystic Endometrial Hyperplasia-Pyometra in White Tiger
}

Shibu Simon', Jacob Alexander², P. Anzeena Hind"1, T. Rajiv³, S. Sooryadas ${ }^{4}$, P.T. Dinesh ${ }^{4}$, P.R. Umashankar ${ }^{5}$, E.K. Easwaran ${ }^{6}$, R. Anoop ${ }^{3}$, A.G. Gouri', V.D. Vijayakumar ${ }^{7}$

10.18805/ag.D-5510

\begin{abstract}
Background: Successful medical management of a serious form of Cystic Endometrial Hyperplasia-Pyometra (CEH-P) in a white tiger, with a combination of mifepristone, misoprostol, cloprostenol, cabergoline and enrofloxacin is discussed.

Methods: A 14-year-old white tiger (Panthera tigris) belonging to the Zoological Garden, Thiruvanathapuram showed symptoms of gradual discomfort, dyspepsia and polydipsia for two weeks. There were no signs of oestrus for the previous two months and a serosanguinous vaginal discharge was also noticed. On ultrasonographic examination, uterine sacculations filled with fluid could be found which was suggestive of pyometra. Leukocytosis, neutrophilia, increased level of serum progesterone, anaemia, high ESR, persistent hyperproteinaemia and hyperglobulinaemia were evident on haematological examination. Considering the risks associated with performing OHE in large felids, medical management was planned. A combination of mifepristone, misoprostol, cloprostenol, cabergoline, enrofloxacin and supportive therapy were given along with strict and close monitoring by different experts on site or by utilizing telemedicine communication technologies. Periodic hematological examination, serum biochemistry, progesterone estimation and ultrasonographic evaluation were done to evaluate the progress of treatment. The progesterone values during the period of study ranged from $45.6 \mathrm{ng} / \mathrm{ml}$ at the commencement of treatment to a basal level of $0.73 \mathrm{ng} / \mathrm{ml}$ towards the end of treatment.

Result: The animal had an uneventful clinical recovery. Considering the risks associated with performing OHE in large felids, medical management can be considered as a valuable option in treating CEH-Pyometra in wild felids. This being the first report of successful medical treatment of CEH-P in a large captive felid with a combination of antiprogestins, prolactin antagonists, prostaglandins and antibiotics, it is suggested that due consideration of such a treatment regimen is of great importance in large felids.
\end{abstract}

Key words: Cabergoline, Cloprostenol, Mifepristone, Misoprostol, Pyometra, Tiger.

\section{INTRODUCTION}

Pyometra can be defined as a hormonally mediated, accumulation of purulent material in the uterus, secondary to bacterial infection, occurring predominantly during the dioestrus stage of oestrous cycle. The hormonal stimulation result in changes in the endometrium including glandular proliferation, hyperplasia and secretion. Pyometra is often seen secondary to cystic endometrial hyperplasia (CEH) probably resulting from chronic progesterone exposure of the endometrium. Secondary bacterial flare-up of normal vaginal flora that enters the uterus during pro-oestrus and oestrus can contribute to the development of CEH (Feldman et al., 2000; Moxon, et al., 2016). Pyometra has been reported in parous and nulliparous large felids mostly over 10 years of age (McCain et al., 2009; Rainey et al., 2018; Palomar et al., 2020). Hematological and biochemical tests can reveal leucocytosis with neutrophilia, anaemia, decrease in the level of albumin, increase in total proteins (globulin), urea and creatinine (Palomar et al., 2020). Anterior vaginal culture is usually taken for demonstration of uterine infection either during proestrus/oestrus or when there is a pathologic discharge from the uterus. Bacterial presence during proestrus and oestrus is common and these are the normal inhabitants of vaginal flora (Kustritz et al. 2006). Use of contraceptives like melengestrol acetate (MGA) when used in zoo felids have deleterious effect on endometrial health
${ }^{1}$ Department of Animal Reproduction, Gynaecology and Obstetrics, College of Veterinary and Animal Sciences, Mannuthy-680 651, Thrissur, Kerala, India.

${ }^{2}$ Zoological Gardens, Dept of Museums and Zoos, Thiruvanathapuram-695 033, Kerala, India.

${ }^{3}$ Govt Multispecialty Hospital, Kudappanakunnu, Thiruvanathapuram695 043, Kerala, India.

${ }^{4}$ Department of Veterinary Surgery and Radiology, College of Veterinary and Animal Sciences, Pookode-673 576, Kerala, India. ${ }^{5}$ Biomedical Technology Wing, Sree Chitra Tirunal Institute of Medical Sciences and Technology, Thiruvanathapuram-695 011, Kerala, India. ${ }^{6}$ Senior Consultant Veterinarian, Thiruvanathapuram-695 011, Kerala, India.

${ }^{7}$ Pilikula Biological Park, Mangalore-575 028, Karnataka, India.

Corresponding Author: Shibu Simon, Department of Animal Reproduction, Gynaecology and Obstetrics, College of Veterinary and Animal Sciences, Mannuthy-680 651, Thrissur, Kerala, India. Email: shibu@kvasu.ac.in

How to cite this article: Simon, S., Alexander, J., Hind, P.A., Rajiv, T., Sooryadas, S., Dinesh, P.T., Umashankar, P.R., Easwaran E.K, Anoop, R., Gouri, A.G. and Vijayakumar, V.D. (2022). A Novel Protocol for Medical Management of Cystic Endometrial Hyperplasia-pyometra in White Tiger. Agricultural Science Digest. DOI: 10.18805/ag.D-5510.

Submitted: 06-10-2021 Accepted: 29-12-2021 Online: 28-01-2022 
(Munson, et al., 2002). Pyometra being a life-threatening disease of domestic and non-domestic animals usually requires ovariohysterectomy (OHE) to save the life of the animal. However, medical management of pyometra has been reported to be successful in domestic canines and felines (Simon et al., 2009; Chinnu, 2016; Ay et al., 2018; Romagnoli, 2018 a,b; Simon, 2019). Although surgical treatment of pyometra in large felids have been reported in tiger, lion, leopard, liger (lion-tiger crossbreed) and jaguar, surgical risks, anaesthesia and postoperative complications have been described (McCain et al., 2009; Rainey et al., 2018; Palomar et al., 2020). Therefore, consideration of medical management is of marked importance in endangered species like tiger. The present study is the first report of a successful medical management of a serious form of Cystic Endometrial Hyperplasia-Pyometra (CEH-P) in a large felid, with a combination of mifepristone, misoprostol, cloprostenol, cabergoline and enrofloxacin along with supportive therapy.

\section{MATERIALS AND METHODS}

A 14-year-old white tiger (Panthera tigris), weighing $130 \mathrm{~kg}$ and housed at the Zoological Garden, Thiruvananthapuram was observed with symptoms of gradual discomfort, dyspepsia, polydipsia and vaginal discharge for two weeks. No signs of oestrus were evident during the previous two months. The animal showed purulent vaginal discharge and microscopical examination of the vaginal smear revealed disintegrated neutrophils (Fig 1). Culture and sensitivity tests performed on the collected pus, did not exhibit growth of any particular bacteria.

On ultrasonographic examination with Mindray Z6 Vet ultrasound machine, uterine sacculations filled with pus could be observed which was suggestive of pyometra (Fig2). The ultrasonographic findings were further supported by the blood picture that revealed leucocytosis $(24,200$ cells/ cu.mm) with neutrophilia (Fig 3), elevated serum progesterone levels $(45.6 \mathrm{ng} / \mathrm{ml})$ (Fig 4), elevated ESR (95-100 mm/h), hyperproteinaemia (peak value $8.4 \mathrm{gm} / \mathrm{dL}$ ) and hyperglobulinaemia (peak value $4.6 \mathrm{gm} / \mathrm{dL}$ ) (Fowler and Muller, 2004).

Considering the risks of surgical interventions in large felids of advanced age, it was decided to adopt a conservative medical management approach. The animal was closely monitored simultaneously by experts on site as well as experts from other parts of the state by utilizing telemedicine communication technologies. Perusal of available literature on treatment protocols for pyometra in large felids revealed the lack of any standardized medical protocols that could be used in such cases. In this context, a medical management protocol that involved the use of antiprogetins, prostaglandins and antiprolactinics was designed for use in large felids with pyometra after carefully considering the action of these drugs in domestic canines and felines. In order to eliminate the chances of potential side effects, if any, a lower dose treatment regimen was used in the initial protocol (Protocol A) from $18^{\text {th }}$ December 2020 (Day 1). As part of the protocol, mifepristone (200 mg tablet) was given per orally once daily (OD) on Days 1 and 2 and subsequently the dosage was increased to twice daily (BID) on Days 3, 4, 8, 15 and 22. Continuous evacuation of

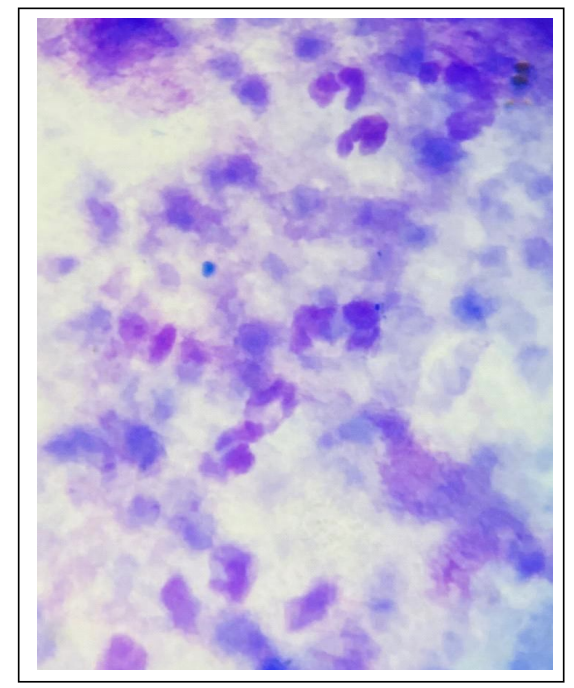

Fig 1: Disintegrated neutrophils observed in the vaginal smear.

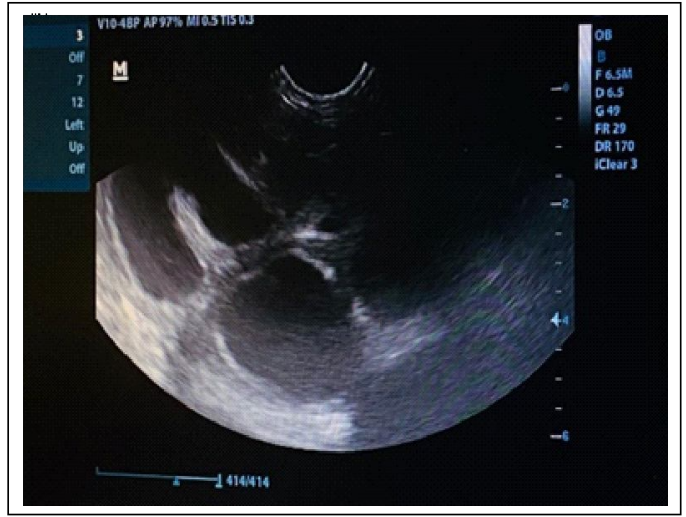

Fig 2: Ultrasonogram showing uterine sacculations filled with anaechoic fluid before commencement of treatment.

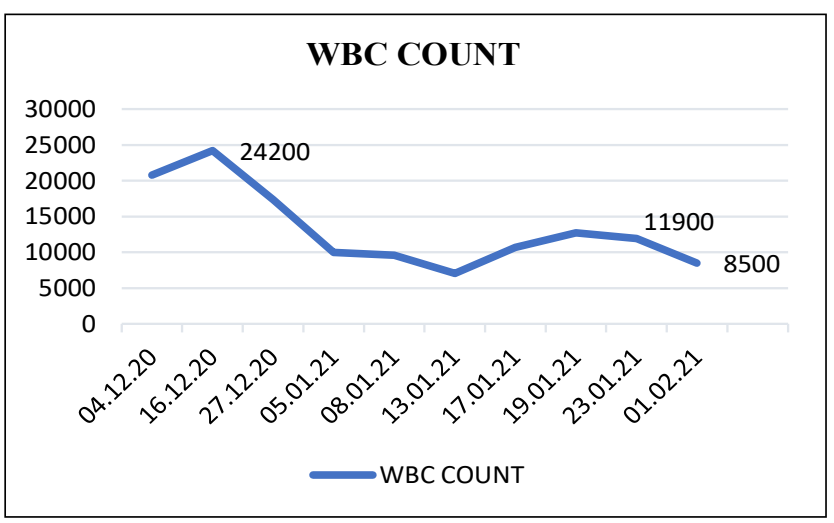

Fig 3: WBC count of the white tiger during the course of the treatment. 
purulent discharge could be observed from $12 \mathrm{~h}$ after the first dose of mifepristone. Toxaemia and associated signs were managed with supportive treatment and a long acting enrofloxacin at $5 \mathrm{mg} / \mathrm{kg}$ intramuscularly at an interval of three days. Condition of the animal improved after the administration of mifepristone and enrofloxacin as evidenced by increased physical activity and improved appetite.

Protocol $A$ also included the administration of misoprostol (200 mcg tablet) per orally OD on Day 4 and after observing no side effects the dosage was increased to $400 \mathrm{mcg}$ BID for the next 8 days (from Days 5 to 12). A gradual reduction in vaginal discharge was noticed and the consistency of the discharge changed from purulent to mucoid. The animal became apparently healthy and active in a few days. Review sonographic examination was performed after a week of treatment and it was observed that the sacculations and fluidity in the uterus had reduced considerably (Fig 5). After the commencement of normal food intake, $0.25 \mathrm{mg}$ cabergoline was given mixed with meat $O D$ orally for 14 days from Days 8 to 21. Blood urea nitrogen (BUN) and serum creatinine levels showed higher values during the last few days of the first protocol (Fig 6,7).

With the experience gained from the first protocol a higher dose of mifepristone and a more potent prostaglandin analogue cloprostenol was included in the subsequent protocol (Protocol B). In the Protocol B, mifepristone was administered at the rate $300 \mathrm{mg}$ BID on Days 24, 25, 26, 33 and 40. From Day 27 onwards, 130 $\mathrm{mcg}$ of Cloprostenol $(1 \mathrm{mcg} / \mathrm{kg}$ ) was given on alternate days for 7 days, subcutaneously in dived doses, after prior SC administration of $2 \mathrm{ml}$ atropine $(0.6 \mathrm{mg} / \mathrm{ml}) 15$ minutes before in order to minimise the possible side effects. Cabergoline at the rate $0.25 \mathrm{mg}$ OD was given for 10 days from Days 28 to 37.

Haematological examination, serum biochemistry, progesterone estimation and ultrasonographic evaluation were done during the entire course of treatment and the animal is being routinely monitored in this regard to evaluate the progress of treatment (Fig 4, 5, 6 and 7).

\section{RESULTS AND DISCUSSION}

Continuous evacuation of serosanguinous discharge was observed $12 \mathrm{~h}$ after first dose of mifepristone. Review scanning after a week showed considerably reduced sacculations and fluidity in the uterus and no uterine sacculations could be observed after the completion of Protocol A. The tiger showed definite signs of improvement by that time. However, blood urea nitrogen (BUN) and serum creatinine levels were showing higher values during the last few days of the first protocol (Fig 6,7). Leukopaenia was also evident in the present report, after the initial leucocytosis during the course of the disease, where the lowest value was observed to be $7040 / \mu \mathrm{L}$ by Day 27 of treatment (Fig 3).
The Protocol B, with a higher dose of mifepristone and a more potent prostaglandin analogue cloprostenol along with cabergoline, showed remarkable improvement in the condition of the animal. The serum progesterone values reached $0.73 \mathrm{ng} / \mathrm{ml}$ within 3 weeks of treatment which

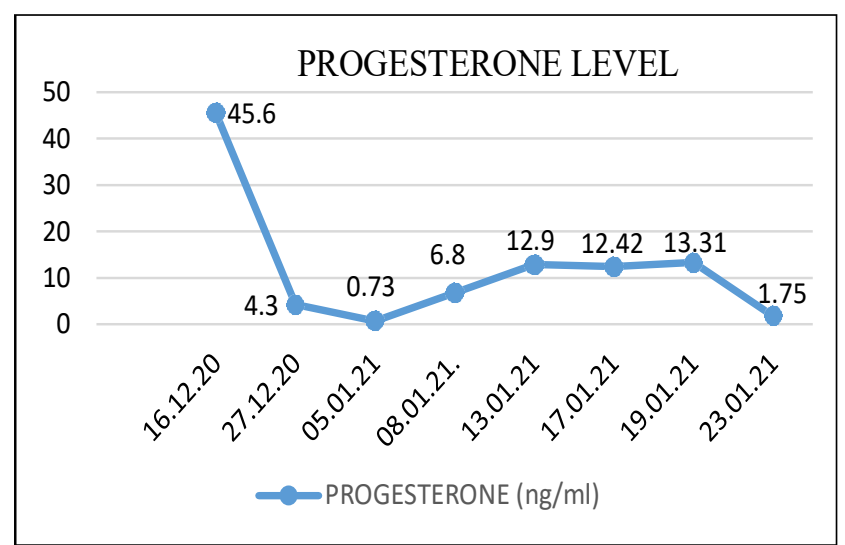

Fig 4: Serum progesterone levels of the white tiger during the course of treatment.

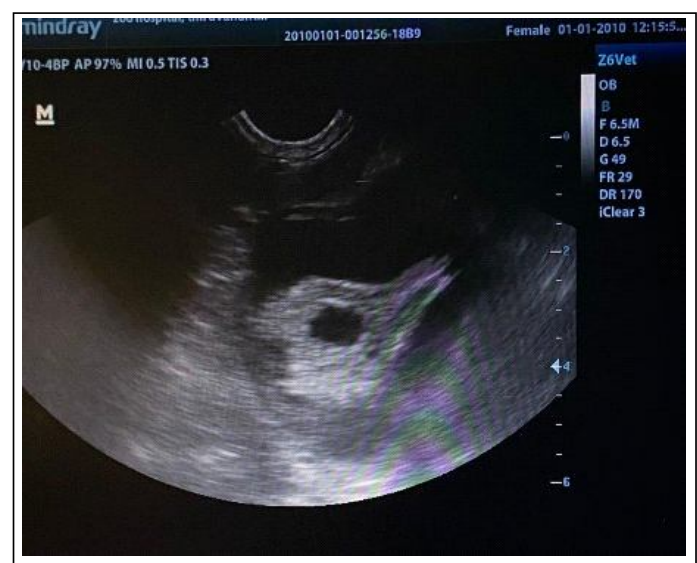

Fig 5: Ultrasonogram showing smaller uterine sacculations filled with reduced quantity of anechoic fluid one week after initial treatment.

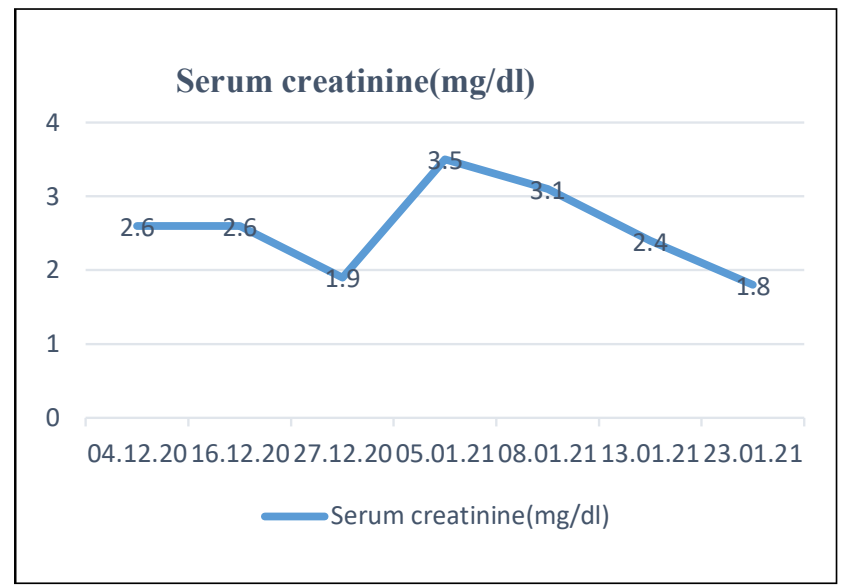

Fig 6: Serum creatinine of the white tiger during the course of treatment. 
probably can be considered as basal value for tiger (Fig 6). When ultrasonography was done after the course of treatment, normal uterine echogenicity with no fluid accumulation could be observed (Fig 8). The animal had an uneventful recovery on completion of the course of treatment.

Pyometra affects large exotic felids especially those aged more than 10 years. Progesterone suppresses the leukocyte response in the uterus, decreases myometrial contractility and stimulates the secretory activity of the endometrial glands during luteal phase of oestrous cycle and predisposing to a medium for secondary bacterial proliferation through the vaginal flora (Guillermo and Richard, 2000). Spontaneous ovulation can occur in lions with varying frequency like domestic cats when kept single or with other females (Schramm et al., 1994), the same appears to be true for the tiger (Graham et al., 2006).

Leukocytosis as observed in the present report on a large felid is also a common finding in domestic carnivores and felines with pyometra (Feldman, 2000). Similarly,

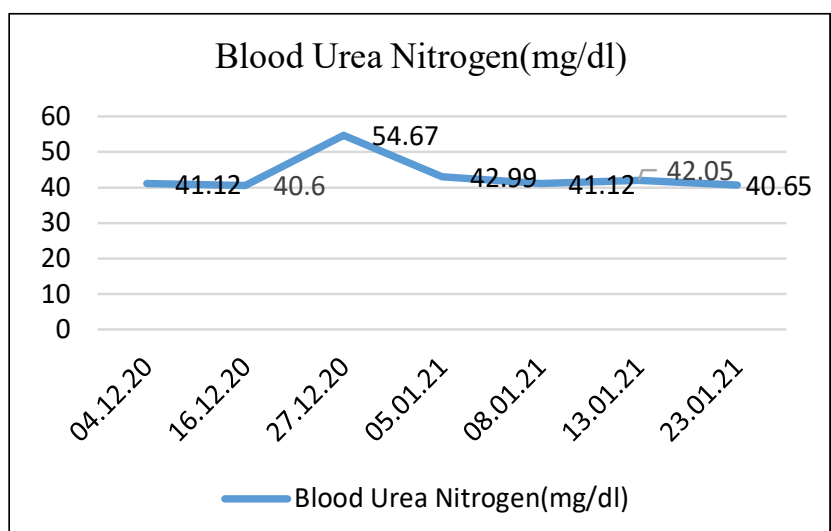

Fig 7: Blood urea nitrogen of the white tiger during the course of treatment.

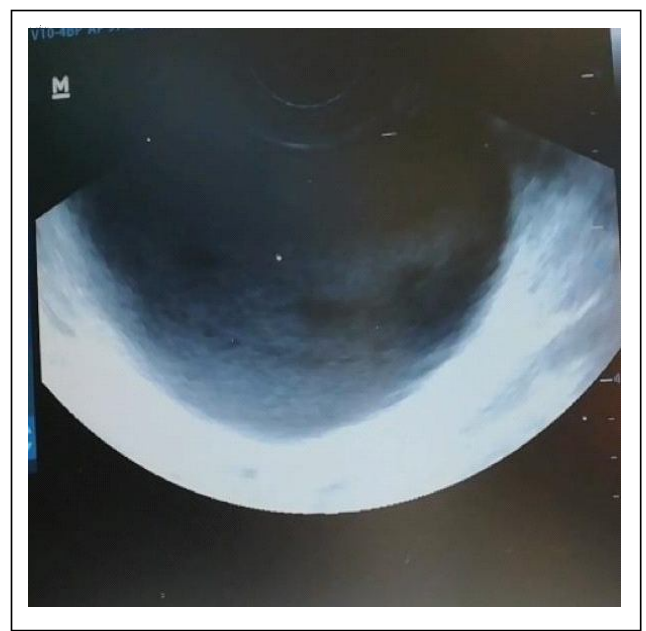

Fig 8: Ultrasonogram after the course of treatment showing normal uterine echogenicity with no fluid accumulation below the anechoic urinary bladder leukopenia as observed in the present tiger was also reported in domestic dogs and cats with pyometra as a result of septicaemia or sequestration of white blood cells into the uterine lumen (Hedlund et al., 2002).

McCain et al. (2009) opined that due to the interplay between the age of the animal and the use of contraceptive implants, cystic endometrial hyperplasia and pyometra are complicated in large felids in captivity. The use of such contraceptives could not be traced in the present report.

Hyperproteinemia and hyperglobulinemia were observed in the present case as usually seen in domestic carnivores with pyometra, presumably due to chronic antigenic stimulation as reported by Feldman (2000).

According to Seal et al. (1985), a single blood sample from tigers which showed elevated progesterone levels cannot be considered as proof of functional luteal tissue or as an indicator of ovulation or pregnancy. There are chances of elevation of progesterone levels above $2 \mathrm{ng} / \mathrm{ml}$ which could be of adrenal origin as a result of handling and immobilization. In another study, Brown et al., (1993) could not detect any relationship between elevated progesterone with cortisol and ACTH in lions. Hence, more research work is needed to explain the physiological significance of this correlation. Ultrasonographic findings were also similar to those described for canines (Chinnu, 2016).

In the present clinical case, we used a combination therapy with mifepristone, misoprostol, cloprostenol, cabergoline and enrofloxacin which was found to be an effective method of medical management in large felids and avoid risks associated with the surgical procedure. Mifepristone (RU 486) is an orally active progesterone receptor site antagonist having high affinity for progesterone receptors (3 times greater than that of progesterone in canines and 9 times in felines). This molecule established safe cervical patency in the present study without any side effects as reported in domestic canines and felines by Simon et al. (2009), Chinnu (2016), Romagnoli, (2018a), Ay et al. (2019) and Simon (2019). Although aglepristone is not currently available in India the use of the same in large felids may further enhance the efficacy of the treatment.

To evacuate uterine contents in bitches and queens with pyometra, a synthetic analogue of PGE1 (misoprostol) with a strong uterotonic action was used initially which was in agreement with Romagnoli (2018a), Simon (2019) and Ay et al. (2019). Misoprostol increases the activity of hyaluronic acid, collagenases elastase and glycosaminoglycan in the cervix and the intracellular calcium levels in the uterus. As a result, it will lead to uterine contractions (Aronsson et al., 2004).

Later a more potent prostaglandin analogue cloprostenol with uterotonic and luteolytic action was used and with the dose used in the present study no side effect other than salivation was noticed. When the same protocol is adapted in future in similar clinical situations in large felids, cloprostenol 
may be administered once the cervix is open so that we can reduce the total duration of the course of treatment or after initial use of misoprostol a shift to cloprostenol can be effectively planned as in the present study.

Prolactin, secreted by the lactotroph cells of the anterior pituitary gland, is a major luteotrophic hormone in the canines and felines. It appears to be an absolute requirement for canine and feline progesterone secretion, by day 30 after ovulation. Hypothalamus possess prolactin inhibiting dopaminergic neurons and are responsible for the major control mechanism of its secretion. Cabergoline is essentially an ergot alkaloid and dopamine agonist. These have strong dopamine D2- receptor agonist activity and can reduce prolactin secretion leading to suppression of its levels. Combination of cloprostenol and cabergoline is more effective in reversing the clinical signs of cystic endometrial hyperplasia (Simon et al., 2009; Chinnu, 2016; Romagnoli, 2018a; Simon, 2019) and possibly the repair of uterine tissue.

\section{CONCLUSION}

Medical management can be considered as a valuable option in treating CEH-Pyometra in large felids. Even when an ovariohysterectomy is planned, initial medical management with the same protocol would ensure surgical fitness of the affected animals. The present protocol should be evaluated in a greater number of animals to standardize the dose requirement and duration of treatment in large felids. Till such findings are available, the authors are of the considered opinion that when medical management is attempted in similar cases, it would be prudent to start treatment with mifepristone and once uterine evacuation process has started to use misoprostol also. After the clinician is satisfied that uterine evacuation is proceeding uninterruptedly, misoprostol may be replaced with closprostenol within a few days. Cabergoline is to be included in the treatment protocol for a minimum of two weeks after ensuring that the animal has regained its appetite. Mifepristone is to be administered at weekly intervals till the serum progesterone values go below 1 $\mathrm{ng} / \mathrm{ml}$. The study also points towards the potential application of ultrasonography in the diagnosis of such reproductive pathologies in large felids.

\section{ACKNOWLEDGEMENT}

The authors are thankful to the Director, Zoological Garden, Thiruvanathapuram and the Honourable Vice Chancellor, Kerala Veterinary and Animal Sciences University for the facilities and support provided.

\section{REFERENCES}

Aronsson, A., Bygdeman, M. and Gemzell Danielsson, K. (2004). Effects of misoprostol on uterine contractility following different routes of administration. Hum. Reprod. 19: 8184.
Ay, S.S., Aslan, S., Onyay, F., Kaya, D., Koldaş, E., Arslan, S.and Findik, M. (2019). Effect of oral misoprostol, alone orin combination with aglepristone, on mid-term pregnancy termination in cats. J. Feline. Med. Surg. 21: 714-722.

Brown, J.L., Bush, M., Packer, C., Pusey, A.E., Monfort, S.L., O'Brien, S.J., Janssen, D.L. and Wildt, D.E. (1993). Hormonal characteristics of free-ranging female lions (Panthera leo) of the Serengeti Plains and Ngorongoro Crater. Reprod. 97: 107-114.

Chinnu, P.V. (2016). Efficacy of medical management and surgical trans-cervical catherization for canine cystic endometrial hyperplasia. M.V.Sc. Thesis. Department of ARGO, College of Veterinary and Animal Sciences, Mannuthy, Thrissur,Kerala. Kerala Veterinary and Animal Sciences University. pp. 1-108.

Feldman, E. C. (2000). The Cystic Endometrial Hyperplasia/ Pyometra Complex and Infertility in Female Dogs. In: Textbook of Veterinary Internal Medicine: Diseases of the Dog and Cat. [Ettinger, S.J. and E.C. Feldman (Eds.)]. (5 $5^{\text {th }}$ Edn.). W. B. Saunders Co. Philadelphia, Pennsylvania. pp. 1549-1555.

Fowler, M. E. and Miller, R. E. (2004). Zoo and Wild Animal Medicine. ( $5^{\text {th }}$ Edn.). Saunders, Elsevier Science, Port Melbourne. pp. 493-494.

Graham, L. H., Byers, A. P., Armstrong, D. L., Loskutoff, N.M., Swanson, W.F., Wildt, D. E. and Brown, J.L. (2006). Natural and gonadotropin-induced ovarian activity in tigers (Panthera tigris) assessed by fecal steroid analyses. Gen. Comp. Endocrinol. 147: 362-370.

Guillermo, C.C. and Richard, N. (2000). Medicina Interna de Animales Pequenos. (2 ${ }^{\text {nd }}$ Edn.) Intermedica, Buenos Aires Argentina. pp. 525-526.

Hedlund, C.S. (2002). Surgery of the Reproductive and Genital Systems. In: Small Animal Surgery. [Fossum, T.W. (Eds.)]. (2nd Edn.). Mosby, Inc., St. Louis, Missouri. pp. 639-644.

Kustritz, M. V. R. (2006). Collection of tissue and culture samples from the canine reproductive tract. Theriogenology. 66 : 567-574.

McCain, S., Ramsay, Matthew C. Allender, M. S., Carlos Souza, and Schumacher, J. (2009). Pyometra in captive large felids: a review of eleven cases. J. Zoo Wild. Med.40: 147-151.

Melandri, M., Veronesi, M.C., Pisu, M.C., Majolino and Alonge, S. (2019). Fertility outcome after medically treated pyometra in dogs. J. Vet. Sci. 2019 Jul; 20(4): e39._http:/ /dx.doi.org/10.4142/jvs.2019.20.e39

Moxon, R., Whiteside, H. and England, G.C. (2016). Prevalence of ultrasound-determined cystic endometrial hyperplasia and the relationship with age in dogs. Theriogenology. 86: $976-980$.

Munson, L., Gardner, I. A., Mason, R.J., Chassy, L.M. and Seal, U.S. (2002). Endometrial hyperplasia and mineralization in zoo felids treated with melengestrol acetate Contraceptives. Vet. Pathol. 39: 419-427.

Palomar, A.R.R., Bermudez, D.B., Palestino, J.F.P., Cuanalo, B.B., Valdez, M.L. and Mancera, A.E.V. (2020). A case report of diagnosis and resolution of pyometra in a Jaguar (Panther onca) with heart disease. Acta Scientific Veterinary Sciences. 2.11: 05-09. 
A Novel Protocol for Medical Management of Cystic Endometrial Hyperplasia-Pyometra in White Tiger

Rainey, B., Singh, A., Valverde, A., Hoddinott, K., Beaufrère, H. Tindal, L. and Smith, D. (2018). Laparoscopic-assisted ovariohysterectomy for the treatment of pyometra in a Bengal tiger (Panthera tigris tigris). Can. Vet. J. 59: 895.

Romagnoli S. (2018a). Practical use of reproductive hormones in dogs and cats. $43^{\text {rd }}$ WSAVA Congress and $9^{\text {th }}$ FASAVA Congress. 25-28 September 2018. Singapore. pp. 512-516.

Romagnoli S. (2018b). Update of clinical use of aglepristone in bitches and queens. $43^{\text {rd }}$ WSAVA Congress and $9^{\text {th }}$ FASAVA Congress. 25-28 September 2018. Singapore. pp. 552-556.

Seal, U.S., Plotka, E.D., Smith, J.D., Wright, F.H., Reindl, N.J., Taylor, R.S. and Seal, M.F. (1985). Immunoreactive luteinizing hormone, estradiol, progesterone, testosterone and androstenedione levels during the breeding season and anestrus in Siberian tigers. Biol. Reprod. 32: 361-368.
Schramm, R.D., Briggs, M.B. and Reeves, J.J. (1994). Spontaneous and induced ovulation in the lion (Panthera leo). Zoo Biol. 13: 301-307.

Simon, S. (2019). Practical Use of Hormones in Canine and Feline Reproduction. In: Compendium XXXV Annual Convention of the ISSAR and International Symposium on Global Perspectives to Enhance Livestock Fertility through Modern Reproductive Techniques for Doubling Farmer's Income; $18^{\text {th }}$ to $20^{\text {th }}$ December,2019. VC and RI, Namakkal, TANUVAS. pp.130-141.

Simon S., Sreejith, J.R. and Ghosh, K.N.A. (2009). Medical management of canine pyometra with mifepristone and prostaglandin. In: Compendium International Summit on advancing medical care: Challenges and strategies: 19, 20 February, 2009, Chennai. pp. 157. 